\title{
Ärztin und Patient in der Zukunft - ein Fall für Zwei?
}

Christine Romann

Korrespondenz:

Dr. med. Christine Romann

Mitglied Zentralvorstand FMH

Fachärztin für Psychiatrie und

Psychotherapie

Feldeggstrasse 12

CH-8008 Zürich

Tel. 044420967

christine.romann[at]bluewin.ch
Unter diesem Titel werde ich an den kommenden «Trendtagen Gesundheit Luzern» (s. Kasten) einen Beitrag leisten zum Thema: Die Patientengeneration der Zukunft.

Wie wird sie denn, diese neue Generation, von der man annimmt, dass sie immer besser informiert und vernetzt sein wird? Ist sie damit auch selbständiger und besser in der Lage, medizinische Entscheide mitzubestimmen, hat sie auch mehr Gesundheitskompetenz und will sie selbstverständlich mehr Eigenverantwortung übernehmen? Solchen Fragen will sich die Tagung widmen, die sich als interdisziplinäre Dialog-Plattform versteht und sich an Meinungs- und Entscheidungsträger im Gesundheitswesen wendet.
Der Patient trifft nun in der ersten Konsultation auf einen Arzt, der im Verlaufe des Gespräches ebenfalls eine Hypothese über die Krankheit des Patienten erstellt, auf der Grundlage seines medizinischen Wissens, manchmal schon nach wenigen Minuten (dass dabei auch beim Arzt Subjektives eine Rolle spielt, soll im Moment hier nicht weiter ausgeführt werden). Der Patient erzählt die Geschichte seiner Erkrankung, der Arzt sammelt Daten - man ahnt, dass es hier zu Missverständnissen kommen kann. Für das Gelingen der Arzt-Patienten-Beziehung und damit für den Verlauf der Erkrankung und ebenso für die Compliance kann das verheerende Folgen haben: Der Patient wird nur solche medizinische Massnahmen mittragen, die

\section{Hat der Patient der Zukunft bessere Krankheitstheorien? Bei einer chronischen Krankheit sogar fundierteres Wissen als die Ärztin?}

Aus unserer Sicht als Ärztinnen und Ärzte möchte ich dazu der Frage nachgehen, was das Verhältnis der beiden - Arzt und Patientin eingeschlossen - kennzeichnet und wie es sich in naher Zukunft weiterentwickeln könnte.

Kommt eine Patientin in meine Praxis - in irgendeine ärztliche Praxis - sucht sie Hilfe. Sie hat oft schon eine längere Suche hinter sich, kommt nun selber nicht weiter und braucht professionellen Beistand. Wir sind gehalten, unser Wissen und Können zur Verfügung zu stellen, damit die Hilfesuchende wieder gesund wird. Doch was sucht sie oder er denn nun wirklich? Patienten bringen nicht nur ihr Leiden mit, sondern in der Regel auch eigenes Wissen über ihre Krankheit, eine eigene Interpretation dessen, was ihnen widerfährt, und persönliche Theorien über mögliche Ursachen. Diese höchstpersönlichen Krankheitsmodelle werden dem allen Menschen eigenen Bedürfnis gerecht, zu verstehen, was uns geschieht, zu fragen, welchen Sinn das haben soll, und schliesslich beinhalten sie auch Vorstellungen, ob wir selber etwas dagegen tun können - die Erkrankung muss im eigenen Leben verortet werden. Solche subjektiven Krankheitstheorien werden in der Regel nicht spontan berichtet, sie spielen aber für den Krankheitsverlauf und für das Arzt-Patienten-Verhältnis eine grosse Rolle. sich mit seinem Konzept der Erkrankung decken - besonders dann, wenn das alles gar nie zur Sprache kommt. Die Tausenden von Tabletten, die Jahr für Jahr im Abfall landen, sind ein beredtes Beispiel für die verpassten Chancen in der Kommunikation zwischen Arzt und Patient.

Hat nun der Patient der Zukunft bessere - für uns Ärztinnen und Ärzte heisst das zunächst: unseren medizinischen Modellen nähere - Krankheitstheorien? Hat er oder sie bereits eine fundierte Auseinandersetzung mit den Symptomen hinter sich, sich ausge-

\section{Die Patientengeneration der Zukunft}

8. Trendtage Gesundheit Luzern

Der Treffpunkt, wenn es um die Zukunft im Gesundheitswesen geht

Mittwoch, 21. und Donnerstag, 22. März 2012

KKL Luzern, Luzerner Saal

Die Trendtage Gesundheit Luzern thematisieren Perspektiven und Trends im Gesundheitswesen. Die Veranstaltung steht wiederum unter dem Motto Machbarkeit - Finanzierbarkeit - Ethik. Programm und Anmeldung unter: www. trendtage-gesundheit.ch 


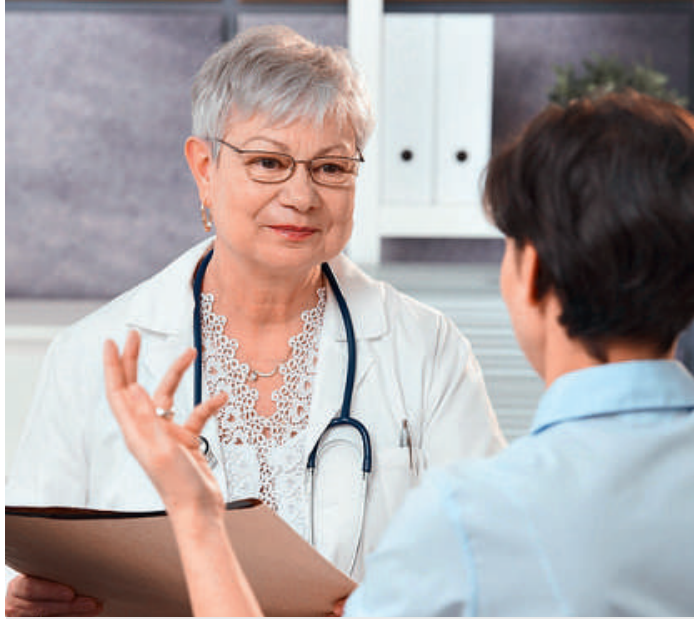

Was macht ein erfolgreiches ärztliches Gespräch aus?

tauscht mit anderen, kommt vielleicht - bei einer seit längerem bestehenden chronischen Krankheit - sogar mit einem fundierteren Wissen als die behandelnde Ärztin selbst in die Konsultation? Oder hat er oder sie sich in den Weiten des Internets auf Irrwege begeben und kommt mit Krankheitstheorien im Kopf, von

\section{«Was hat Ihrer Meinung nach zu Ihrer Erkrankung geführt?»}

denen die behandelnden Ärzte in der Regel keine Ahnung haben und deshalb nicht verstehen, von was die Patienten eigentlich reden? Daten sammeln wird zum Hindernislauf.

Das, wo auch immer, verfügbare medizinische Wissen alleine wird nicht dafür sorgen, dass aus den beiden ein gutes Team wird. Aber es kann sehr wohl dazu beitragen. Dass entsprechende Angebote im Internet bereits heute rege benutzt werden, weist auf ein wachsendes Bedürfnis auch gesunder Menschen hin, sich zu orientieren und auf die eigene Gesundheit einwirken zu können. Zu diesem Wissen gehört mehr und mehr auch eine Schulung im Umgang mit Ärztinnen. Gesundheitskompetenz heisst auch, die Sicht des ärztlichen Gegenübers antizipieren zu können und die benötigte Information in nützlicher Frist so zu liefern, dass Ärzte etwas damit anfangen können. Ein sehr gutes Beispiel hierfür ist ein Flyer des Schweizerischen Seniorenrates: ein Leitfaden, der helfen soll, sich auf den Arztbesuch vorzubereiten. Wer sich vorbereitet auf den Arztbesuch, hat weitaus grössere Chancen, zu den Informationen zu kommen, die er braucht - weil er eben viel eher die Informationen liefert, die der Arzt braucht. «Stellen Sie kurze, klare Fragen und stellen Sie nur eine Frage auf einmal» ist am Schluss in einem Kästchen vermerkt. Ist das nicht sehr klug?

Auch wir Ärztinnen und Ärzte sind gefordert. Vergessen wir nicht, dass das vormalige Wissensmonopol auch ein Machtmonopol war. Nun gilt es, zu partnerschaftlicher Zusammenarbeit zu finden - ohne zu verleugnen, dass die Beziehung nie ganz symmetrisch sein wird.

Was macht ein erfolgreiches ärztliches Gespräch aus? Worauf ist zu achten, wenn wir in einem Erstgespräch die Grundlagen schaffen wollen für eine tragfähige Arzt-Patienten-Beziehung? Und wie verändert sich das, wenn Patientinnen uns auf Augenhöhe begegnen? Natürlich ist das Gesundheitswissen, wie das allgemeine Wissen auch, in der Gesellschaft ungleich verteilt - nicht jeder hat schon einen CrashKurs über seine Symptome im Internet hinter sich. Aber für alle gilt: Unser Gegenüber ist ein Mensch mit eigenen Vorstellungen über seine Krankheit, über sich selber, über seinen Platz auf dieser Welt. «Was hat Ihrer Meinung nach zu Ihrer Erkrankung geführt?», ist eine der möglichen Fragen, die die Welt des Patienten entschlüsseln kann und ihn dort abholt, wo er sich mit seinen Ängsten, seinen Erwartungen und Hoffnungen befindet. Fühlt der Patient sich verstanden, ist er zur Kooperation bereit. Findet er sich gehört, teilt er sich auch mit. Wird er ernst genommen, übernimmt er auch Verantwortung.

So können der kranke Mensch und seine Ärztin sich gemeinsam auf den Weg machen - mit verteilten Rollen - aber auch mit dem Wissen, dass beide Rollen wichtig sind. 